\title{
Development of a Neuro-fuzzy MR Image Segmentation Approach Using Fuzzy C-Means and Recurrent Neural Network
}

\author{
Dipankar Ray ${ }^{1}$ and D. Dutta Majumder ${ }^{2,3}$ \\ 1 Department of CSE, Indian School of Mines, Dhanbad - 826 004, India \\ dpnkray@yahoo.co.in \\ 2 Institute of Cybernetics and Information Technology, Kolkata - 700 108, India \\ ddmdr@hotmail. com \\ 3 Indian Statistical Institute, Kolkata - 700 105, India
}

\begin{abstract}
A neuro-fuzzy clustering framework has been presented for a meaningful segmentation of Magnetic Resonance medical images. MR imaging provides detail soft tissue descriptions of the target body object and it has immense importance in today's non-invasive therapeutic planning and diagnosis methods. The unlabeled image data has been classified using fuzzy c-means approach and then the data has been used for training of an Elman neural network. The trained neural net is then used as a ready-made tool for MRI segmentation.
\end{abstract}

Keywords: Medical Image Segmentation, Neuro-Fuzzy, Elman Recurrent Neural Network (ERNN).

\section{Introduction}

Medical image segmentation provides the basis for any kind of high-level image analysis and subsequent therapeutic and diagnostic planning. Medical images in general contain considerable uncertainty, unknown noise, limited spatial resolution, poor contrast and non-uniform intensity distribution leading to difficulties with segmentation [1]. Among them noise removal is a challenging task and makes segmentation approach unstable. Segmentation on the basis of spatial information can smooth out acquisition noise as well as reduce classification ambiguities. Here we have proposed a semiautomatic segmentation strategy incorporating cognitive computational ability of two well-known soft computing approaches, namely, fuzzy and neural network (NN) models, that is purely data-driven and self organized approach. It is done primarily by identifying the characteristic properties of the unlabeled image feature space composed of gray levels of each voxel; then by an artificial neural network (ANN) with the labeled feature space. The information obtained from the unsupervised fuzzy clustering is used to train the subsequent supervised clustering approach. This is achieved by applying a fuzzy c-means approach as unsupervised clustering (UC) and a recurrent neural network model as supervised clustering (SC). Dynamically 

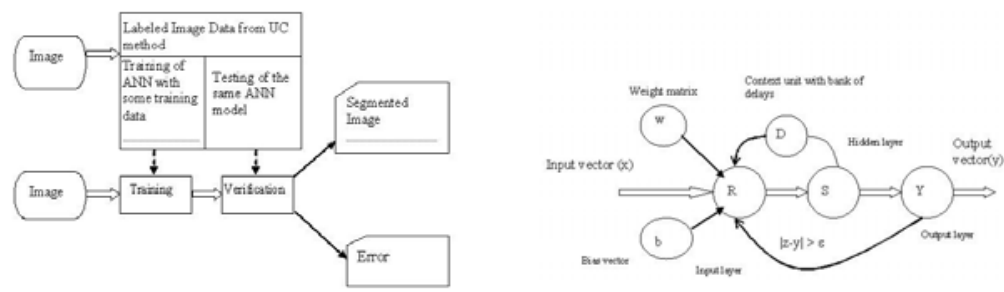

Fig. 1. (a) DFD and Process flow diagram of the segmentation approach and (b) State flow diagram of a recurrent neural network

driven recurrent neural structures can capture both the temporal as well as spatial information distribution patterns making it a suitable device to represent state-space configuration for dynamic nonlinear systems and adaptive prediction of function parameters. The Levenberg-Marquardt (LM) algorithm is used as the backpropagation (BP) optimization strategy of the used recurrent network. The method is basically a nonlinear least square minimization algorithm with a blend of gradient descent and Gauss-Newton iterative methods. The outcomes of the segmentation were visually verified and compared with predefined pathologically tested segmented data by practising radiologists.

\section{Fuzzy Segmentation of MR Images}

In a fuzzy segmentation, each pixel is assigned a membership value for each of the $c$ regions. This forms a matrix $U$ that partitions the image. Considering the membership values of each pixel we often obtain more accurate estimates of region properties. But this approach assigns pixels to more than one region and regions do not preserve the connectivity property of segmentation which "tear up tissue classes" into medically incorrect regions. This problem can also be solved either by hardening each column of $U$ with suitable hardening function [2] or by assigning fuzzy colors to each pixel by mixing $c$ basic colors in proportion to their memberships. Colors can be selected as per the subject matter of the image or by some pre-established expectations in the medical community.

\section{Unsupervised Fuzzy Clustering}

Fuzzy clustering can handle the uncertainty and imprecision better than the deterministic or statistical approaches. Among the various fuzzy soft computing approaches, fuzzy c-means (FCM) model and algorithm family is most popular and well-developed least square model. A generalized model of optimization can be defined in terms of an objective function $\mathrm{J}_{m}$ as [2]:

$$
\underbrace{\min }_{(u, v)}\left\{J_{m}(U, V, w)=\sum_{i=1}^{c} \sum_{k=1}^{n} u_{i k}^{m^{\prime}} d_{i k}^{2}+\sum_{i=1}^{c} w_{i} \sum_{k=1}^{n}\left(1-u_{i k}\right)^{m^{\prime}}\right\}
$$


Where $\mathrm{U}=\left[\mathrm{u}_{i k}\right]$, a $c \times n$ matrix, is the $c$-partition of the object data $X=$ $\left\{x_{1}, \ldots, x_{n}\right\} \subset R^{P}, \mathrm{~m}^{\prime} \geq 1$ is a measure of fuzziness, $\mathrm{d}_{i k}$ is a Euclidian distance function and $u_{i k}^{m^{\prime}}$ is the membership measure of $\mathrm{k}^{\text {th }}$ data object to $\mathrm{i}^{\text {th }}$ cluster. $V=\left(v_{1}, v_{2}, \cdots v_{c}\right) \in R^{c p} ; v_{i} \in R^{p}$, is the centroid vector of the clusters and $w=\left(w_{1}, w_{2}, \cdots w_{c}\right)^{T} ; w_{i} \in R^{+}$, is the weighting vector and for FCM $w_{i}=0 \forall i$. The distance between $x_{k}$ and cluster centre $v_{i}$ can be expressed as:

$$
d_{i k}=d\left(x_{k}-v_{i}\right)=\left(\sum_{j=1}^{m}\left(x_{k j}-v_{i j}\right)^{2}\right)^{1 / 2}
$$

in m space. For FCM, membership value $u_{i k}$ and the centre $v_{i j}$ can be expressed as:

$$
\begin{gathered}
u_{i k}^{(r+1)}=\left(\sum_{j=1}^{c}\left(\frac{d_{i k}^{(r)}}{d_{j k}^{(r)}}\right)^{\frac{2}{\left(m^{\prime}-1\right)}}\right)^{(-1)} \forall i, k \text { for } I_{k}=\Phi, u_{i k}^{(r+1)}=0 \forall i, i \in I_{k} \\
\text { and } v_{i j}=\frac{\sum_{k=1}^{n} u_{i k}^{m^{\prime}} x_{k}^{j}}{\sum_{k=1}^{n} u_{i k}^{m^{\prime}}} \forall i, m^{\prime}>1, \sum_{i \in I_{k}} u_{i k}^{(r+1)}=1
\end{gathered}
$$

With a suitable guess of the initial partition matrix and a convergence limit $\epsilon_{L}$ the above expressions of $\mathrm{u}$ and $\mathrm{v}$ can be solved using iterative optimization technique as suggested by $\left[3\right.$. To monitor the singularity of the denominator, $d_{j k}^{(r)}$ , of the membership function $u_{i k}^{(r+1)}$ the method proposes a bookkeeping system $I^{k}$ and $\overline{I^{k}}$ to keep a track of null values of $d_{j k}^{(r)} \cdot I_{k}=\left\{i \mid 2 \leq c<n ; d_{i k}^{r}=0\right\}$ is the set of null distance from the $\mathrm{k}^{\text {th }}$ feature point to the cluster centers and $\overline{I_{k}}=\{1,2, \cdots c\}-I_{k}$.

\section{The Recurrent Neural Network}

A schematic representation of the state-space model of a batch recurrent neural network (RNN) model is depicted in Fig. 1(b). The hidden layer neurons constitute the state of the network and the output of the hidden layer is fed back to the input layer through context unit. The input of the model consists of inputs from the given pattern and the context units. Following this general configuration of dynamic behaviour of recurrent network, with $R^{m}$ and $R^{p}$ as input and output space, may be described by the following two equations:

$$
\begin{gathered}
a(t+1)=f\left(w_{h}^{a} x_{t}+w_{c}^{b} a_{t}+b_{h}\right) \\
y(t)=g\left(w_{y}^{a} a_{t}+b_{y}\right)
\end{gathered}
$$

where $w_{h}^{a}:(m \times n)$ is a synaptic weight matrix of the hidden layer $S_{(n \times 1)}$ and input layer $R_{(m \times 1)}: X=\left\{x_{i}: i=1 \cdots m\right\}$. Bias vectors $b_{h}:(n \times 1)$ and $b_{y}$ : 
$(p \times 1)$ are connected to the hidden layer and the output layer respectively. Input layer $R_{(m \times 1)}$ provides $X_{m}$ input in conjunction with $a_{(t-1)}$ from the context unit to the hidden layer. $w_{c}^{b}:(m \times n)$ is the synaptic weight matrix of $\mathrm{n}$ neurons of the hidden layer and is connected to the feedback signals from the context unit. Linear layer $Y_{(p \times 1)}$ with synaptic matrix $w_{y}^{a}:(n \times p)$ and bias $b_{y}$ generates desired output $\mathrm{y}(\mathrm{t})$. The process function $f:\left(R^{m} \rightarrow S^{n}\right)$ is a diagonal mapping of state-space into itself for some memoryless component-wise nonlinearity $f$ : $(R \rightarrow R)$. The measurement function $g:\left(S^{n} \rightarrow Y^{p}\right)$ is a linear mapping of $S^{n}$ space to output space $Y^{p}$.

\subsection{Training Procedure}

The training procedure of the ERNN [4] is implemented by transfer functions (f, $\mathrm{g})$, free parameters $(\mathrm{w}, \mathrm{b})$ and backpropagation optimization strategy. All of these form the basis for the approximation of a mapping function $\mathrm{f}: \mathrm{R}^{m} \rightarrow \mathrm{R}^{p}$, and $\mathrm{x} \rightarrow \mathrm{y}=$ $\mathrm{f}(\mathrm{x})$ for a training data set $X_{m}=\left\{\left(x_{i}, y_{i}\right), i=1 \cdots m\right\}$, where $\mathrm{n}$ is the number of sample pattern. While selecting these parameters, the generalization of the trained network with respect to the unknown test data was kept in mind and selected accordingly. As a transfer function we have used hyperbolic tangent sigmoid function (f) and linear function (g). The tangent sigmoid function can accept any value between plus and minus infinity and can transform them into the range -1 to +1 . To have a fast and memory efficient error back propagation training method we have used Levenberg-Marquardt nonlinear least square minimization algorithm with a memory reduction factor of magnitude 2. Given a set of training samples $\{x(k), y(k) 1 \leq k \leq n\}$ the error backpropagation training begins by feeding all $\mathrm{n}$ inputs through the multilayer neural network model and computing the corresponding output $\{z(k) 1 \leq k \leq n\}$. Then a sum of square error can be written as:

$$
\begin{gathered}
S(w)=\sum_{k-1}^{k}(y(k)-z(k))^{2} \\
=\sum_{k-1}^{k}\left(y(k)-\left(f\left(x(k) w_{h}(k) b_{h}(k)\right)+g\left(h(k) w_{y}(k) b_{y}(k)\right)\right)\right)^{2}
\end{gathered}
$$

where $w_{h}$ and $w_{y}$ are weightage vectors corresponding to the $\mathrm{k}^{\text {th }}$ input vector. In each iteration $w_{h}$ and $w_{y}$ are replaced by a new estimation $\left(w_{i}+\delta\right): i=h, y$ and $\delta$ is approximated by Levenberg-Marquardt optimization method.

\subsection{Optimization Method Using LM Algorithm}

As an optimization technique we have used the Levenberg-Marquardt algorithm as it outperforms simple gradient descent and other conjugate gradient methods in a wide variety of problems. It can be seen that simple gradient descent and Gauss-Newton optimization are complementary in the advantages they provide. Levenberg algorithm is based on this observation and is written as:

$$
w_{i+1}=w_{i}-(H-\mu I)^{-1} J^{T} e
$$


where Hessian matrix $\mathrm{H}$ is evaluated at $w_{i}$ and $e$ is the vector of network errors. The Jacobian matrix contains first derivatives of the network errors with respect to the weights and biases:

$$
J(w)=\frac{\delta e_{j}}{\delta w_{i}} \text { where } 1 \leq j \leq p \text { and } 1 \leq i \leq m
$$

for a performance function $\mathrm{f}: \mathrm{R}^{m} \rightarrow \mathrm{R}^{p}$ defined as $f(x)=1 / 2\|e(x)\|^{2}$. When $\mu$ is zero, the algorithm is just a newton's method and when large, this shows gradient descent behaviour with very small step size. To speed up the convergence in the direction of small gradient Marquardt replaced the identity matrix with the diagonal of $\mathrm{H}$ (Eq. 11). This incorporates a large movement along the directions of smaller gradient.

$$
w_{i+1}=w_{i}-(H+\mu \operatorname{diag}|H|)^{-1} J^{T} e
$$

where $\mu$ is the learning rate. Since $H$ is proportional to the curvature, $w_{i}$ will have a large step in the direction with low curvature and small step in the direction with high curvature [5]. The Levenberg-Marquardt function is used with a memory reduction factor of two by dividing Hessian as a linear combination of two Jacobians $J_{1}$ and $J_{2}$ :

$$
H=J_{1}^{T} J_{1}+J_{2}^{T} J_{2}
$$

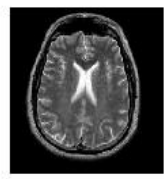

(a)Original MRI Image
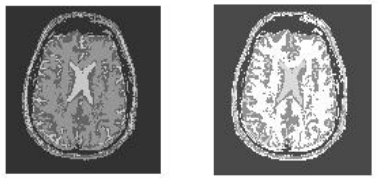

(b)FCM Segmentetion (c)EIman ANN Segmentetion

Fig. 2. Original MRI (a) with FCM (b) and Elman NN (c) segmented images

\section{Experiments and Results}

The study has been done on transaxial MR-T2 images (Fig. 2(a)). The image is a $256 \times 256 \times 3$ RGB image with voxel dimension $1 \times 1 \times 3 \mathrm{~mm}^{3}$. The image has been taken from the Harvard Medical School "the whole brain image atlas" image database. Initially, image pixels are (visually) classified into four clusters namely GM, WM, CSF and non-brain matter (cranium and mandible). At the outset we have randomly selected a number of pixels and divided them into training data set and checking data set. The training data set pixels are first classified into four classes by an unsupervised fuzzy clustering (UC) technique. Then the data points are divided into four regions or classes and assigned appropriate membership values corresponding to each of the four classes. Model is trained using a randomly selected $n$ number of training and membership values to each 
class. This simulation process continues by adjusting different NN parameters till the model reaches the required given accuracy level. After successful convergence, the trained NN engine is verified with the check-data set and their corresponding classes. Once the NN is ready, it can be used to determine the membership values of any input data from the given test images. Visually, the accuracy can be verified by comparing the membership graph of the NN model and the UC model but to confirm the desired accuracy level, a regression analysis has been done between the network response and the corresponding targets. The correlation coefficient we get is almost a unity. In the comparative study of Elman, Back Propagation and Radial Basis Function [6] neural network models, we have found that in a normalized environment Elman model takes least number of epochs (olny 12 compared to 244 and 125 epochs of BP and RBF respectively) to reach the given goal of accuracy and the simulation is also within the acceptable range (Fig. 2(c)).

\section{Conclusion}

In this article a neuro-fuzzy model has been proposed to deal with the MR medical image segmentation. This model can provide a robust and stable segmentation solution over the intrinsic uncertainty of any MR image. The SC model performs segmentation on the basis of trained activation function with the help of labeled data as identified by the unsupervised method. Quantitative performance and visual comparisons clearly demonstrate the superiority of the combined method. Furthermore, we have found that this proposed segmentation model does not require image preprocessing as the supervised model trains up the activation function with a very large number of labeled data that minimizes the chance of influence of noise components and inhomogeneity, if any, in the image.

\section{References}

1. Ray, D., Dutta Majumder, D.: Studies on Some multimodal medical image registration approaches for diagnostics and therapeutic planning: With some case studies. Science Letters, NAS 28(5\&6), 135-154 (2005)

2. Sutton, M.A., Bezdek, J.C., Cahoon, T.C.: Image Segmentation by Fuzzy Clustering: Methods and Issues. In: Bankman, I.N. (ed.) Handbook of Medical ImagingProcessing and Analysis, pp. 87-106. Academic Press, London (2000)

3. Bezdek, J.C., Hathaway, R.J., Sabin, M.J., Tucker, W.T.: Convergence theory for fuzzy C-means: counter examples and repairs. IEEE Trans. Syst. Man Cybern. 17873-17877 (1987)

4. Elman, J.L.: Finding structure in time. Cognitive Science 14, 179-221 (1990)

5. Hagan, M.T., Menhaj, M.: Training feedforward networks with the Marquardt algorithm. IEEE Transaction on Neural Networks 5(6), 989-993 (1994)

6. Powell, M.J.D.: Radial basis functions for multivariable interpolation: a review. In: Algorithms for Approximation, pp. 143-167. Clarendon Press, Oxford (1987) 